\title{
Profile and Outcome of Adult Spine Pathologies Managed in a Neurosurgical Tertiary Care Center in Nepal
}

\author{
Hemant K Sah, Dipendra K Shrestha, Binod Rajbhandari, Gopal Sedain, Amit B Pradhanang, Sushil \\ K Shilpakar, Mohan R Sharma \\ Department of Neurosurgery, Maharajgunj Medical Campus, Tribhuvan University Teaching Hospital, Institute \\ of Medicine, Kathmandu, Nepal
}

\section{Corresponding author:}

\section{Mohan R Sharma, MBBS, MS}

Department of Neurosurgery, Maharajgunj Medical Campus, Tribhuvan University Teaching Hospital, Institute of Medicine, Kathmandu, Nepal.

Email:mohanrajsharma@gmail.com

Submitted : Mar 2, 2020

Accepted : Apr 7, 2020

\begin{abstract}
\section{Introduction}

Spine pathology involves a wide spectrum of diseases and needs a multidisciplinary approach including surgery, rehabilitation and psychological support. It increases the burden to the family and society. This study describes diseases related to spine and spinal cord from a neurosurgical department of a tertiary hospital in Nepal.
\end{abstract}

\section{Methods}

This is a retrospective study of all spinal cases admitted between April 2019 to February 2019, in the Department of Neurosurgery, Tribhuvan University Teaching Hospital, Kathmandu, Nepal. All adult patients of $\geq 16$ years, diagnosed with various spinal diseases were included. Based on the spectrum of causative pathologies, the study population was broadly categorized into trauma, degenerative, tumor, vascular and infection. Demographics, other variables, and outcome at 1 month were assessed.

\section{Results}

A total of 71 patients were included in this study. Male preponderance was observed in all categories. Twenty three patients (32.4\%) had traumatic spine injury and fall from height was the most common mode of injury, accounting for two-third of the total spinal trauma patients. Cervical segment was involved in two-third patients. More than one-third of the spinal patients (36.6\%) had a degenerative disorder involving lumbar (57.7\%) and cervical regions (42.3\%). Spinal infection was diagnosed in $11.2 \%$ of the patients with $62.5 \%$ diagnosed as Pott's spine. Overall complications were seen in $20 \%$. Pain improved in all patients while overall good outcome was noted in $63.2 \%$ of the patients.

\section{Conclusion}

Our study demonstrated a large patient burden and a clinical profile dominated by preventable causes such as RTA and fall injury. With early treatment and rehabilitation, significant improvement can be achieved. Further large scale multicenter studies are required to generalize the findings of this study to the whole population of Nepal.

Keywords: Burden, Nepal, spine trauma, spine pathology 


\section{INTRODUCTION}

Sa pine pathology involves a wide spectrum of disease and needs a multidisciplinary approach including surgery, rehabilitation, and psychological support. The burden of spine disease is well described in a review article by Filip et al. ${ }^{1}$ It increases the burden because it involves the cost of care during the hospital stay and treatment and also limits the working limit of an individual posttreatment. ${ }^{1}$ Hence, access to appropriate treatment is crucial. ${ }^{2}$ In low and middle-income countries (LMICs) such as Nepal, there has been a rapid increase in spine trauma which is probably due to the rapid motorization over 2 decades. ${ }^{3}$ Excessive speed, driving under the influence of alcohol, and poor road design and infrastructure-related factors are some major risk factors for the increasing number of road traffic accidents (RTA). Deaths per 100,000 populations are 15.9 in Nepal. ${ }^{4}$

Average age for a patient with spinal disease, especially traumatic spinal cord injuries (TSCls) is between 37 and 47.9 years. ${ }^{4-6}$ Male are at higher risk for TSCls. ${ }^{3,5,6}$ It is probably due to factors exposed due to outdoor activities. Spinal cord injury (SCI) results in severe compromise to neurologic structures, resulting in possible paralysis, sensory loss, bladder or bowel dysfunction, and mortality. ${ }^{6}$ Most common cause is attributed to fall injury in LMICs. ${ }^{5,6}$ Other spinal pathology includes tumor, vascular and degenerative changes. The aim was to retrospectively investigate the overall profile and outcome of a spectrum of spinal pathologies admitted to the Department of Neurosurgery of our institute over 11 months.

\section{METHODS}

This is a retrospective observational study, conducted at Tribhuvan University Teaching Hospital (TUTH), which included patients admitted from April 2019 to February 2020, in the Department of Neurosurgery. All patients of age $\geq 16$ years and with spine disease were included and were categorized into different groups, namely trauma, degenerative, tumor, vascular, and infection. Ethical approval was obtained from the Institutional Review Committee of the Institute of Medicine, Nepal. All patients were treated as per the protocol of the Department of Neurosurgery, TUTH. A pro forma was used to collect the data. The variables included patient demographics (age, sex), mode of injury, American Spinal Injury Association (ASIA) neurology at admission for trauma, symptoms, diagnosis, histopathology, management (surgery/ conservative/referred), postsurgery hospital stay, and outcome (complications and mortality within 30 days). Statistical analyses were performed using Stata version 21.0 (Stata Corp, College Station, Texas). Descriptive statistics were used to describe patient characteristics.
Table 1. Categories of spine pathology

\begin{tabular}{|c|c|c|}
\hline Categories & & Number (\%) \\
\hline Traumatic & & $23(32.4 \%)$ \\
\hline Degenerative & & $26(36.6 \%)$ \\
\hline Tumor & & $11(15.5 \%)$ \\
\hline Infective & & $8(11.3 \%)$ \\
\hline \multirow[t]{2}{*}{ Vascular } & & $3(4.2 \%)$ \\
\hline & Total & 71 (100\%) \\
\hline
\end{tabular}

\section{RESULTS}

A total of 71 patients were admitted in the Department of Neurosurgery during this period. Spine and spinal cord disease constituted 21\% of the cases operated in the Department of Neurosurgery. Seventy-one patients were treated. Surgical management was done in 63 patients while 8 patients were managed conservatively. Fifty five cases (77.5\%) were males and 16 (22.5\%) were females, accounting for male-to-female ratio of 3.2:1. Male preponderance was noted in all groups. Age ranged from 16 to 82 years with a mean of $42.5 \pm 16.1$ years. All these cases were categorized into five groups as shown in Table 1. Majority of the spinal cases belonged to traumatic and degenerative groups (69\%) while there were only three cases with vascular pathology.

In regards to presenting clinical manifestation, the patients with sensory (tingling and numbness), motor (weakness and sphincter disturbance) and both (motor and sensory) symptoms were 23 $(30.3 \%), 25(32.9 \%)$ and $16(21.1 \%)$, respectively. Pain was present in $44.7 \%$ of patients. Reviewing the causative pathology of spinal cases, twentythree patients $(32.4 \%)$ had been treated for diagnosis of traumatic spine injury. In regards to the mode of injury, fall from height was noted in 18 cases $(78.2 \%)$, followed by RTA in 5 cases $(21.8 \%)$. In spinal injury cases, when probing the level of injury, the cervical segment was involved in 16 $(69.6 \%)$ cases, followed by $3(13.0 \%)$ in lumbar and $4(17.4 \%)$ in thoracic level. On classifying based on the type of spinal injury, subluxation was the most common (47.8\%) followed by burst fracture (26\%). Odontoid fracture was seen in 3 patients and one patient was operated for atlantoaxial dissociation (AAD). When taking into consideration of the severity of the injury at presentation, all spinal injury patients were categorized based on ASIA neurology $A, B, C, D$, and $E$, which were $9(39.1 \%), 1$ (4.3\%), 4 $(17.4 \%), 4(17.4 \%)$ and $5(21.8 \%)$ respectively. Details of the involved segments and the associated type of injury are summarized in Table 2.

Burst fracture was mostly seen in the lumbar and thoracic region while subluxation occurred mostly in the cervical region as shown in Table 2. For spinal instability, posterior instrumentation was done in 16 cases $(25.4 \%)$ and posterior wiring in 2 
Table 2. Spine segment with different nature of trauma

\begin{tabular}{|c|c|c|c|c|c|}
\hline $\begin{array}{l}\text { Segment } \\
(n=23)\end{array}$ & $\begin{array}{l}\text { AAD* } \\
(n=1)\end{array}$ & $\begin{array}{l}\text { Burst } \\
\text { fracture } \\
(n=6)\end{array}$ & $\begin{array}{c}\text { Compression } \\
\text { fracture } \\
(n=2)\end{array}$ & $\begin{array}{l}\text { Odointoid } \\
\text { fracture } \\
(n=3)\end{array}$ & $\begin{array}{l}\text { Subluxation } \\
\qquad(n=11)\end{array}$ \\
\hline $\begin{array}{l}\text { Cervical } \\
(n=16[69.6 \%])\end{array}$ & 1 & 1 & 1 & 3 & 10 \\
\hline $\begin{array}{l}\text { Thoracic } \\
(n=4,[17.4 \%])\end{array}$ & 0 & 2 & 1 & 0 & 1 \\
\hline $\begin{array}{l}\text { Lumbar } \\
(n=3,[13.0 \%])\end{array}$ & 0 & 3 & 0 & 0 & 0 \\
\hline
\end{tabular}

*AAD-atlantoaxial dissociation

(3.2\%). For degenerative compressive myelopathy, decompression was performed in 5 patients $(7.9 \%)$. For $A A D$, posterior interlaminar wiring was done. Cervical burst fracture was treated with anterior corpectomy and fusion in one case) and posterior instrumentation in four cases. Three patients with traumatic spine injury (one with burst fracture, one with compression fracture, one with subluxation who refused for surgical intervention were discharged on request. Details of surgical procedure performed for trauma and degenerative patients are shown in Table 3.

Altogether 11 patients $(16.0 \%)$ had tumor of the spinal cord, nerve sheath or spine; and the final histopathology report is summarized in Table 4. Gross total resection (GTR) was achieved in 8 cases $(72.8 \%)$, partial resection in $2(18.2 \%)$ and subtotal in $1(9.0 \%)$.

Twenty-six patients with degenerative spine disorder were admitted and involved levels were lumbar region in 15 cases (57.7\%), followed by the cervical region in 11 cases (42.3\%). Prolapsed intervertebral disc (PIVD), ossified posterior longitudinal ligaments (OPLL), and lateral recess stenosis (LRS) were seen in 22 (84.6\%), 3 (11.5\%), and $1(3.8 \%)$ patients, respectively. Pain was present in $45 \%$ of the patients. Microdisectomy was done in 13 cases. Two patients were managed conservatively for PIVD.

Table 3. Approaches used for the treatment of spine pathology $(n=63)$

\begin{tabular}{lc}
\hline \multicolumn{1}{c}{ Procedure } & Number (\%) \\
\hline Posterior instrumentation & $16(25.4 \%)$ \\
Microdisectomy & $13(20.6 \%)$ \\
Anterior cervical discectomy and fusion & $8(12.7 \%)$ \\
Posterior decompression & $5(7.9 \%)$ \\
Anterior cervical corpectomy and fusion & $2(3.2 \%)$ \\
Brooks' methods (interlaminar wiring) & $2(3.2 \%)$ \\
360 Fixation & $1(1.6 \%)$ \\
Others (infective and vascular & $16(25.4 \%)$ \\
pathology) &
\end{tabular}

Vascular spine pathology was found in only 3 (3.9\%) patients. One patient with intradural extramedullary arteriovenous malformation (AVM) and one patient with vertebral body hemangioma were treated surgically. Vertebral body hemangioma was treated with posterior instrumentation and injection of absolute alcohol. One patient who was previously embolised was referred for re-embolization.

A total of 8 patients $(10.5 \%)$ diagnosed with spine infection were treated and among them $5(62.5 \%)$ were of Pott's spine. Among them, 6 cases underwent surgery for decompression and biopsy. Antitubercular drugs (ATT) for Pott's spine and antibiotics for other infective cases were given. Out of 6 , one case was operated by anterior cervical corpectomy and fusion while 2 cases with Pott's spine were posteriorly instrumented and decompressed while in other cases posterior decompression was done with drainage of pus. Two cases were managed conservatively with antibiotics and analgesics as both patients were neurologically intact and did not have any signs of instability. The treatment strategic scheme that was given to the patients is summarized in Table 5.

Conservative management was done with Sternooccipital-mandibular immobilization (SOMI) brace, Philadelphia collar, antibiotics, analgesics, and ATT as required in remaining patients. Mean postsurgery hospital stay is $10 \pm 6.2$ days (range 1-30 days). One patient was hospitalized for 182 days and died from

Table 4. Histopathology report of tumor patients $(n=11)$

\begin{tabular}{lc}
\hline \multicolumn{1}{c}{ Histopathology } & Number (\%) \\
\hline Schwannomas & $3(3.9 \%)$ \\
Astrocytoma (WHO I) & $2(2.6 \%)$ \\
Meningioma (WHO I) & $2(2.6 \%)$ \\
Lipoma & $1(1.3 \%)$ \\
Ependymoma (WHO II) & $1(1.3 \%)$ \\
Ewing sarcoma & $1(1.3 \%)$ \\
Epidermoid cyst & $1(1.3 \%)$ \\
\hline
\end{tabular}


Table 5. Treatment scheme based on different causative spinal pathology

\begin{tabular}{lccccc}
\hline \multicolumn{1}{c}{ Treatment } & $\begin{array}{c}\text { Traumatic } \\
(\mathrm{n}=23)\end{array}$ & $\begin{array}{c}\text { Degenerative } \\
(\mathrm{n}=26)\end{array}$ & Tumor $(\mathrm{n}=11)$ & Infective $(\mathrm{n}=\mathbf{8})$ & Vascular $(\mathrm{n}=3)$ \\
\hline Surgery $(\mathrm{n}=63)$ & 19 & 25 & 11 & 6 & 2 \\
Referred $(\mathrm{n}=1)$ & 0 & 0 & 0 & 0 & 1 \\
Conservative $(n=7)$ & 4 & 1 & 0 & 2 & 0
\end{tabular}

* discharged on patient's request

pneumonia leading to sepsis. This was only one patient who was in hospital stay for such a long time so this was treated as an outlier and was removed from the calculation of mean.

Complications were observed in 14 patients, accounting for an overall complication rate of $20 \%$. Out of 14 cases with complications, one patient developed quadriplegia and chest infection, subsequently succumbed due to full-blown sepsis. Overall chest infection was seen in 4 patients $(5.6 \%)$ and $28.6 \%$ among all complications. One patient developed discitis who was successfully managed with antibiotics and analgesics alone without any need for surgical exploration. One patient who was operated for Ewing sarcoma with intraspinal extension had recurrence. Other complications were trivial such as transient deranged Liver function test and increase in urea and creatine and transient increase in pain, which resolved with medical and non-surgical treatment.

Forty-eight patients (63.2\%) showed improvement in the chief complaints they were admitted for. In the case of degenerative spine disease, improvement was seen in most of the patients. Only 5 out of 23 patients had no improvement in power. All patients showed improvement in pain. In traumatic spine injury improvement was seen in 9 (39.1\%) patients only. Those with ASIA A and B neurology showed no improvement, and only 1 out of 3 with ASIA C neurology showed improvement. Three out of 4 patients with ASIA D neurology showed improvement in power and pain.

\section{DISCUSSION}

Male preponderance is seen in all groups, mainly in trauma and degenerative groups which are probably because males do outdoor work in LMICs. ${ }^{5}$ Spine trauma is common in LMICs where there is poor development of roads and the economy is based on agriculture. The geography is such that the roadways culminate in fall injury. ${ }^{5}$ This is contrary to papers published from developed and developing countries. ${ }^{2}$

The mean age was 42 years similar in other paper. ${ }^{5}$ Previous studies have described only a part of spine disease such as spinal cord injury ${ }^{7}$ and spine trauma published from Nepal.5,8 In this study, we analyzed the spine and spinal cord disease by dividing into 5 categories i.e. trauma, degenerative, infection, tumor and vascular groups. In our study, traumatic spine injury was the leading cause, similar to a study from South Africa. ${ }^{2}$ In traumatic spine injury, the most commonly involved segment is the cervical spine, (65.2\%) in our study. ${ }^{5}$ About 30\% of the patient presented with neurological deficit with most of them was with ASIA A neurology (39.1\%). Treatment modality included conservative (SOMI brace, immobilization), surgical approach (ACDF, posterior instrumentation and wiring), and combined anterior plus posterior instrumentation as required.

Degenerative spine disease was the second most common cause of spine disease constituting $36.6 \% .^{2}$ Pain in our patient was present in $45.0 \%$ of the cases which is lower than in a study by Fillip et al. ${ }^{1}$ PIVD is one of the most common cause for nerve root compression. ${ }^{9}$ Treatment modalities used were open microdisectomy for PIVD. There are microendoscopic approach ${ }^{9}$ and percutaneous transforaminal endoscopic discectomy ${ }^{10}$ and a newer concept like only fixation technique. ${ }^{11}$ Posterior laminectomy and decompression for OPLL involving multiple segments was done.

Infection of the spine was seen in $11.3 \%$ cases only and among these Pott's spine was seen in $62.5 \%$ of the patients. This finding is consistent with similar studies in LMICs. ${ }^{2,12}$ However, bacterial infection occurs in larger numbers in other countries ${ }^{2}$ and the Pott's spine cases are increasing in developed countries. ${ }^{12}$ Average postsurgery hospital stay was 10 days which was much shorter than previously reported from eastern Nepal (31 days). ${ }^{5}$ Tumor operated were mostly nerve sheath tumors like schwannoma, and intramedullary tumors like astrocytoma, lipoma and ependymoma, and others in decreasing frequency. ${ }^{13}$

\section{CONCLUSION}

Our study describes different spine disease from a tertiary neurosurgical unit in Nepal and demonstrated a large patient burden and a clinical profile dominated by preventable causes such as 
RTA and fall injury. Patient with spine trauma as well as other diseases need multidisciplinary treatment. Post-treatment, patients are not efficient to work for quite some time which increases the burden on family and society.

\section{CONFLICT OF INTEREST}

None declared.

\section{REFERENCES}

1. Raciborski F, Gasik R, Kłak A. Disorders of the spine . A major health and social problem. Reumatologia. 2016;54(4):196-200.

2. Miseer S, Mann T, Davis J. Burden and profile of spinal pathology at a major tertiary hospital in the Western Cape, South Africa. SA Orthop J. 2019;18(1):33-9.

3. Aleem IS, DeMarco D, Drew B, et al. The burden of spine fractures in India: A prospective multicenter study. Glob Spine J. 2017;7(4):325-33.

4. WHO. Global Status Report on Road. World Health Organization. 2018;20. Available from: https:// www.who.int/violence_injury_prevention/road_ safety_status/2018/en/

5. Shrestha D, Garg M, Singh GK, et al. Cervical spine injuries in a teaching hospital of eastern region of Nepal: A clinico-epidemiological study. J Nepal Med Assoc. 2007;46(167):107-11.
6. Ning $G Z, W u Q, L i Y L$, et al. Epidemiology of traumatic spinal cord injury in Asia: A systematic review. J Spinal Cord Med. 2012;35(4):229-39.

7. Prakash Kafle, Babita Khanal, Dipak Kumar Yadav, et al. Spinal cord injury, Clinical Profile and its Management at Tertiary Care Center in Nepal. J Nobel Med Coll. 2019;8(14):16-21.

8. Devkota P, Manandhar H, Khadka P. Spinal Injuries in a Tertiary Care Referral Center of Western Nepal. Nepal J Med Sci. 2013;2(2):156-9.

9. Yadav RI, Long L, Yanming C. Comparison of the effectiveness and outcome of microendoscopic and open discectomy in patients suffering from lumbar disc herniation. Medicine (Baltimore). 2019;98:50.

10. Chen Z, Zhang L, Dong J, et al. Percutaneous transforaminal endoscopic discectomy compared with microendoscopic discectomy for lumbar disc herniation: 1-year results of an ongoing randomized controlled trial. J Neurosurg Spine. 2018;28(3):300-10.

11. Goel A. Prolapsed, herniated, or extruded intervertebral disc-treatment by only stabilization. J Craniovertebr Junction Spine. 2018;9(3):133-34.

12. Garg RK, Somvanshi DS. Spinal tuberculosis: A review. J Spinal Cord Med. 2011;34(5):440-54.

13. Schellinger KA, Propp JM, Villano JL, et al. Descriptive epidemiology of primary spinal cord tumors. J Neurooncol. 2008;87(2):173-9. 